\title{
Energy Efficient Cluster based Routing Protocol for Wireless Sensor Networks
}

\author{
N. Thangadurai \\ Research Scholar, Research \& Development Centre, \\ Bharathiar University, Coimbatore, India.
}

\begin{abstract}
Wireless sensor network is a great boon to wireless technology which can be used in various critical applications, making it more familiar in emerging technologies which we use in our day to day life. Even though it has several advantages, it has some drawbacks like limited communication bandwidth, energy consumption etc. The purpose of the paper is to design an energy efficient cluster based routing protocol to minimize energy consumption since energy resource is the major life factor for a node. Usage of clustering concept in hierarchical protocol provides more advantages than any other traditional routing protocols. LEACH and LEACH-C are most commonly used hierarchical routing protocols. The author is proposing an enhancement of LEACH-C protocol, instead of using constant round time usage of adaptive variable round time method provides a multi-hop communication between distance nodes to base station. Thus by using this method, this protocol can be used for larger geographical region with less energy consumption and less cluster head death. The results were obtained by using NS2 simulator which shows the improvement of overall network efficiency by comparing with existing protocols.
\end{abstract}

\section{Keywords:}

LEACH, Mobile Collector, Variable Round Trip Time

\section{INTRODUCTION}

Sensor networks are becoming a major technology because of its sensing application and process the data to the user at regular time periods. This phenomenon is used in many fields such as industrial applications, military applications, home applications, agriculture and medical applications. But these sensing applications are battery oriented [1]. Thus the minimum usage of energy prolongs the network lifetime. But in some applications it's very critical to change the battery if it totally drained off, which leads to network failure or the messages cannot be reached to the user. More energy is needed when the node is transferring data to user. Keeping all this in mind, many routing protocols have been designed. Hence for an efficient routing protocol it must consume less energy for routing the data to the user. Some of the major characteristics of WSN are [2]: 1. Network Lifetime-In WSN, nodes uses limited power supply by using batteries, which is difficult to replace in certain environment. Alternatively using solar cells provides recharging of batteries in such environment. But this is not effective enough, so energy requirement is the major factor in WSN; 2. Fault Tolerance-In WSN the nodes may run out of energy or damaged through environment changes. So it must tolerate to all such conditions. For overcoming this redundant deployment of nodes should be done by scalability. Since the nodes can be deployed in larger geographical regions, so the protocols must be scalable depending upon the architecture; 3 . Programmability- If a task is completed; the nodes must have the flexibility to change their task by developing a new program. Hence ways of processing information is the main advantage in WSN; 4. MaintainabilityThe nodes must maintain their own health status by monitoring its conditions like depleting batteries or damage etc. ; 5.Quality of Service-WSN applications must be tolerant for latency,

\author{
R.Dhanasekaran,Ph.D \\ Director-Research, Syed Ammal Engineering \\ College,Ramanathapuram, India.
}

reliable detection of events, thus the quality approximation increases the quality of service.

\section{EXISTING ROUTING MODELS}

There are three types of routing protocols [3, 4] available for WSN: 1) Flat based routing, in which all nodes are typically assigned equal roles or functionality. 2) location-based routing, the positions of sensor nodes are exploited to route data in the network 3) Hierarchical routing protocols, which is based on cluster architecture i.e. the sensing environment is divided in two groups which is called as clusters. In each cluster there will be a cluster head, which collects the data from nodes and aggregates this data to the user or base station. These clustering techniques have following merits: High scalability, Re-use of bandwidth, Decreases communication transparencies and Improves resource sharing, Provides inter and intra cluster communication, Reduces communication overhead. Thus hierarchical routing protocols are having more advantages when compared to other routing protocols. The main types of hierarchical routing protocols are LEACH and LEACH-C. The existing research work shows some important hierarchical based protocols, which provides efficient energy consumption because of clustering architecture.

\subsection{LEACH}

Low energy adaptive clustering hierarchy is a routing protocol in WSN which uses cluster based architecture and multi hop communication. Here the nodes are grouped together to form clusters which contains cluster head and this is selected by using distributed algorithm. Its main function is to aggregate the data to base station. LEACH uses a TDMA/CDMA MAC to reduce inter-cluster and intra-cluster collisions. LEACH has two operational phases: one is set up phase and another is steady state phase [13]. Formation of cluster head and cluster is done in set up phase and selects itself as a $\mathrm{CH}$ randomly, a predetermined fraction of nodes $\mathrm{p}$ elect themselves as CH's. Here node chooses a random number, between 0 and 1 . If this random number is less than a threshold value, T (n), then the node becomes a clusterhead for the present round. This threshold value is calculated by using parameters like the desired percentage to become a cluster-head, the present round, and the number of nodes not been selected as a cluster-head in the last $(1 / \mathrm{P})$ rounds, and it is denoted by a term $\mathrm{G}$. Where the term $\mathrm{G}$ is a node set that involved in the $\mathrm{CH}$ election. [14]

Once $\mathrm{CH}$ is elected it broadcast an advertisement message to the remaining nodes in the network and informs them that it is the new $\mathrm{CH}$ for the current round, after receiving this advertisement the non-cluster head members decides to join the cluster or not. This is mainly based on the signal strength of the advertisement message. Then the non $\mathrm{CH}$ nodes send a join request to the $\mathrm{CH}$, that they will be a member of the $\mathrm{CH}$. Once the $\mathrm{CH}$ receives these messages, based on the number of messages received it forms the cluster, then it creates a TDMA schedule and assigns time slot for each node that when it can transmit. This is been broadcasted to all non-cluster members in the cluster.

Steady State Phase sensor node senses the data and transmits the data to cluster head and then goes to the sleep mode. After receiving all the data the cluster head aggregates this data to the base station. Thus the duration of steady state phase is longer than the duration of setup phase in order to minimize the overhead. Then the network goes back again into setup phase 
again and starts another round of selecting new $\mathrm{CH}$. Each cluster uses different CDMA codes, which reduces the interference from nodes belonging to other clusters and increases the life time of the network. But still there are some disadvantages like it is not applicable to networks deployed in larger regions, this is because LEACH assumes that all nodes can transmit with enough power to reach the base station with the support of different MAC protocols. Then the elected Cluster heads will be concentrated in one part of the network. So this makes some nodes to do not have any vicinity of cluster head, dynamic clustering brings extra overhead like head changes and broadcasting advertisement messages. This overhead problem reduces the energy consumption and it also assumes that all nodes begin with the same amount of energy, assuming that cluster head also consumes same amount of energy as each node.

\subsection{LEACH-C}

In LEACH-C, the formation of cluster is done by using central control algorithm. This produces better clusters by spreading the $\mathrm{CH}$ throughout the network. Thus LEACH-C uses centralized clustering algorithm. Here steady state phase is identical as LEACH [14].

In setup phase stage, the node in the network sends their information about energy, location to the base station and it ensures that all nodes energy is evenly distributed. It calculates its average node energy level. So the nodes which are less than this level cannot become $\mathrm{CH}$ for the current round. So for the remaining nodes it uses an annealing algorithm to find the $\mathrm{K}$ optimal clusters.

This algorithm minimizes the energy consumption of non-cluster head nodes during data transmission to $\mathrm{CH}$. Finally when the $\mathrm{CH}$ is selected by the base station it broadcasts this $\mathrm{CH}-\mathrm{ID}$ of the node to the network. If this ID matches to the particular node then that node will be denoted as a $\mathrm{CH}$ for particular round. The remaining nodes determines TDMA slot for transmission and goes of sleep when no transmission in a network [15]. The advantage of LEACH-C is $\mathrm{CH}$ selection is based on energy level which minimizes the failure of $\mathrm{CH}$. So life time of the network will increase.

The uneven clustering of problem in sensing environment this leads to overload energy consumption problem. Due to uneven clustering some clusters will be maximum sized clusters and some will be minimum sized clusters. This minimum sized cluster will transmit more number of frames to cluster head when compared to maximum sized cluster since it has less member nodes so this makes the cluster head always busy in aggregation of data to base station. Thus it consumes more amount of energy and since in LEACH-C the round time is decided during initialization of network and it is kept as constant because at starting of round every node will have high energy. So the minimum sized cluster head which spends more amount of energy will leads to cluster head death, if it happens between the rounds the members nodes will transmit frames without knowing about the death of the cluster head this will leads to wastage of energy. So the round time is kept constant until the completion of particular round wastage of nodes energy takes place or it also leads to a network failure in which nodes die due to lack of energy.

\section{PROPOSED MECHANISM}

In this paper, the author proposes a new cluster based routing protocol called as VR-LEACH which is an enhancement of LEACH-C protocol which is used to stabilize the power utilization problem of various sensor nodes and to minimize the overload energy consumption problems [16]. Fig.1 Shows VRLEACH uses variable round time which depends upon the minimum cluster size $[15,17,18]$. Normally the network life time is divided into various rounds, and each round starts with the setup stage as like in LEACH-C, whereas every node transmits or sends its identifier Id, position through GPS and also the residual power to the sink or Base Station (BS). Then Base Station (BS)

Divides the network into different optimal clusters before transmitting the cluster data to various sensor nodes and the Base Station (BS) decides the round time to be used for the current round which is called as Tcurrent [19]. Thus to stabilize the overload power utilization problem, the proposed VR-LEACH protocol uses an adaptive round-control technique. In which the round time Tcurrent is defined at the beginning of a current round and Rcurrent that mainly depends on the minimum cluster size and the maximum cluster size.

The frame time Fmin for the cluster Cmin which is having the minimum size that is minimum number of nodes Mmin, is calculated (Eq. 1) as follows:

Fmin $=$ Mmin $* \sigma+\lambda$ (Eq. 1)

Where, $\lambda$ is time required to send data to base station and $\sigma$ constant time slot.

After knowing the frame time we can calculate the present round time Tcurrentis as follows:

Tcurrent $=$ NFavg. Fmin (Eq. 2)

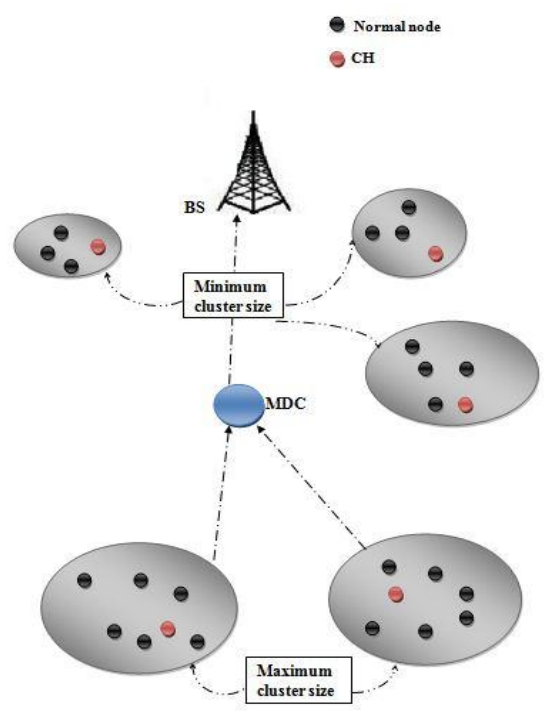

Fig 1: VR-LEACH Architecture

Where, NFavg is the average number of frames for a cluster with the size N/k. Thus from the above equation, $\mathrm{CH}$ of the minimum sized cluster must not transmit frames more than the average number of frames NFavg for the entire round which will reduce the overload power utilization of the Cluster Head of the minimum sized cluster head Cmin, but this is only for the minimum sized cluster the remaining clusters in the network can transmit or send more frames than the average number of frames NFavg.

After describing the round time Tcurrent for the present round, the sink or Base Station (BS) transmits or sends this information to the clusters and also the broadcasts Tcurrent to all the sensor nodes within the network. Therefore, each of the nodes will decide its cluster and also its time period in the particular TDMA program and it will begin the steady state stage. The steady state stage is identical as in existing LEACH-C protocol. Above defined round time will be a variable round time which plays a vital role for energy consumption [20]. Suppose if the round time is kept as large, the operations will be long which makes cluster heads to drain more energy because a cluster head has to work for a long time by transmitting data to the base station from each cluster. Or else if the round time is kept short, nodes will consume more energy in ways of re-clustering such as frequent changes of cluster head transmitting advertisement messages which leads to consumes more energy so both ways it has problems. So the round time should be chosen taking care of the above trade-offs. 
LEACH-C uses a constant round time for nodes in which it calculates round time based upon the initial number of nodes and fixes it for whole network lifetime. But the sensor networks are dynamic in nature that is nodes can be added to the network or some nodes can die due to lack of energy. In case of constant round time, if more nodes are added in the network after round time is decided, then the round time will not be sufficient to complete the operation because of inclusion of new nodes and frequent changes in re-clustering will increase the overhead of network. In other way, if nodes are not alive, then the round time is kept as long enough and this leads to consumption of more energy from cluster heads which does not have enough nodes [21].

Hence constant round time makes the network load unbalanced.so to balance the network, variable round time is used dynamically to adjust based upon the network dimensions. The nodes with very less amount of energy and a selected cluster head for a particular round may not have enough amount of energy to complete the particular round. Because at the beginning, the calculated round might be long enough because while starting every nodes will have high energy. As it process, energy will be drained which leads to the failure of cluster head which finally leads to loss of cluster data. So round time should be adaptive to network. In this paper, the calculated round time mainly depends upon the number of active nodes with its residual energy level in the network. But as network processes, the energy level in each nodes goes down, and based upon the energy level if the round time decided is very small, then it results in frequent re-clustering. So when the numbers of active nodes are very less than the round time, then it is fixed as half of the last round time. By using this method the round will be completed avoiding frequent re-clustering. Thus this approach makes the clustering algorithm more adaptive to network changes. In the beginning, every network is assigned with random and uniformly distributed nodes. For the calculation of round time, the total number of active nodes is calculated. After completion of round time calculation, clustering of nodes is done i.e. cluster head selection, cluster formation, TDMA scheduling. Data communication from nodes to base station via cluster head is processed until round time is not over. The network will end after the death of all nodes as in traditional protocols like LEACH-C which uses single hop communication. So these protocols cannot be used for larger regions. In order to overcome this, we use a $\mathrm{T}$ mobile agent, called a Mobile Data Collector (MDC) [22] this moves between network and collects the data from the nodes. This also helps in data processing, data aggregation and other functions etc. The normal function of MDC moves between the networks periodically and collects the data from nodes and return to the sink to dump the data. These protocols can be used in larger geographical region sensor networks with the MDC architecture, which reduces the energy consumption of the sensor nodes and increases traffic received at base station. It has a multi-hop communication for data aggregation and transmission from sensor nodes to base station with minimum energy consumption [23]. This approach enhances the network scalability for large scale environmental applications. After the cluster formation $\mathrm{CH}$ set up, the Time Division Multiple Access (TDMA) schedules every node to send data towards $\mathrm{CH}$. Then MDCs transmit a beacon message to all $\mathrm{CHs}$ to upgrade their current position. When MDC's received the data from any $\mathrm{CH}$, it will direct the data towards the base station $[24,25]$.

\subsection{Algorithm}

The results were observed by using ns 2 stimulator. Generally in ns2 there are three main files in which ".tcl." is considered as the most important file, contains all node parameters such as area, interface queue length, propagation channel, antenna, number of nodes etc. Another main file is "c.p" (i.e) connection pattern its function is to provide an efficient communication between nodes and finally "S.C" (i.e) scenario pattern, its main function is to make connectivity between nodes. Here C.P is named as cluster 1 and in S.C setting a GOD file is done. In order to generate graph in ns2, AWK format is used. Figure.6 shows the flow of VRLEACH.

Step 1: Parameter values: Parameter values have been defined at first. The parameters such as Sensing Area, Network size, Propagation model, Network interface, MAC interface, Seed, Interface queue type, Interface queue length, Antenna type, transport layer protocol, Application, Stop, MDC energy level, $\mathrm{RX}$ power, TX power, packet were defined at the beginning of the program.

Step 2: Global variables defined: The values of each parameter such as syntax, creation of topography for nodes and nodes position are defined.

Step 3: Trace object for NS and NAM defined: Trace object is created here which are named as trace and NAM trace. Normally this file act as a history for the running program and in trace definition we define topology of $\mathrm{X}$ and $\mathrm{Y}$ for area.

Step 4: Create GOD: It is called as general operational directory whose main function is packet forwarding and receiving, enqueue and de-queues. Here it stores the information about all the nodes so this makes easier for routing between nodes.

Step 5: Global node setting: Configuration of parameters is done in NAM window and bandwidth of MAC layer is also done and nodes are assigned to corresponding channels.

Step 6: Traffic model is defined: For movement of nodes C.P and S.C file is to be loaded. This file is already defined in TCL but now for movement of nodes this file are to loaded using puts command

Step 7: Stimulation ends: The end of stimulation time for nodes to stop communication is allocated here.

Step 8: Defining the graph values and execution of graph: Here defining of graph is done here $\mathrm{X}$ value and $\mathrm{Y}$ value for $(\mathrm{X}, \mathrm{Y})$ axis is defined according to movement of nodes. AWK format is used for generation of graph.

Step 9: Printing XY values and run: The author prints the values of Tracefd, number of nodes, routing, CP, seed, propagation and antenna by using puts command. Then the nodes start the stimulation and command is created for running the program.

\section{SIMULATION RESULTS}

Table.1 Shows parameters assigned to simulate the WSN Environment.

\begin{tabular}{|l|l|}
\hline PARAMETERS & VALUE \\
\hline Sensing Area & $1000^{*} 1000$ \\
\hline Network size & 36 nodes \\
\hline Propagation model & Propagation/two ray ground \\
\hline Network interface & Phy/wirelessphy \\
\hline MAC interface & Mac 802.11 \\
\hline Seed & 0.0 \\
\hline Interface queue type & Queue/DropTail/PriQueue \\
\hline Interface queue length & 7777 \\
\hline Antenna type layer & Antenna/Omni Antenna \\
\hline $\begin{array}{l}\text { transport } \\
\text { protocol }\end{array}$ & FTP \\
\hline Application & $20.0(\mathrm{~s})$ \\
\hline Stop & $1000 \mathrm{~J}$ \\
\hline MDC energy level & $1.0 \mathrm{Watt}$ \\
\hline RX power & $1.0 \mathrm{Watt}$ \\
\hline TX power & Clustering \\
\hline Packet &
\end{tabular}




\subsection{PERFORMANCE EVALUATION}

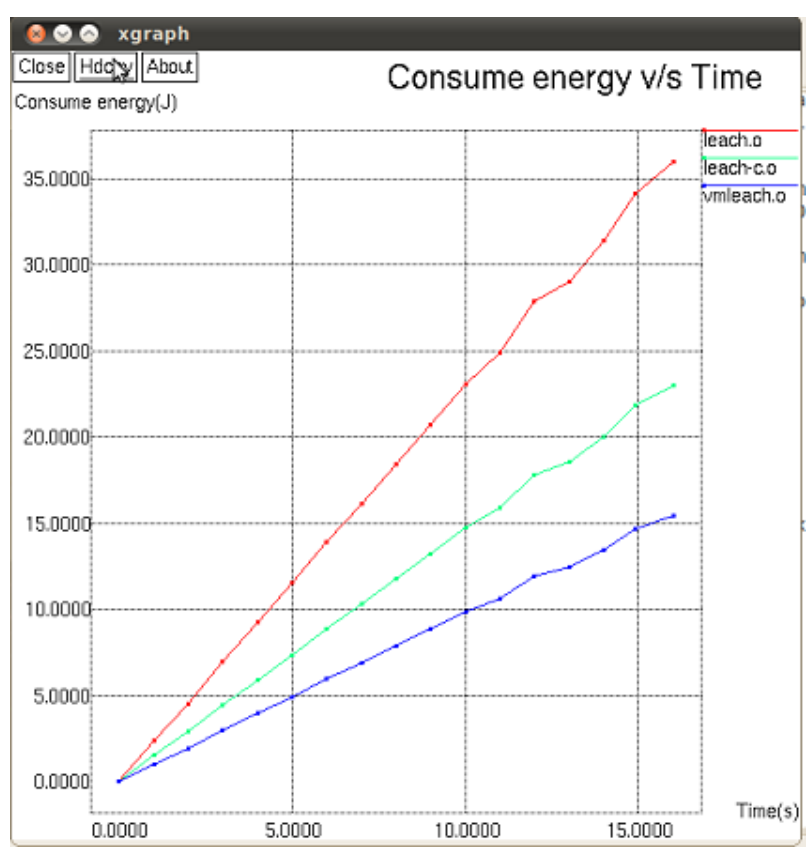

Fig 2: Energy Consumption Vs Time

Fig.2 Shows the simulation result shows the comparison between existing routing protocols LEACH and LEACH-C with VRLEACH. For sensor nodes the crucial thing is minimum energy consumption. The comparison between existing protocols with VR-LEACH. As time increases the energy consumption between the nodes increases. But our proposed mechanism shows less consumption of energy for data transmission by comparing with other protocols as time progress.

\section{QQ $\mathrm{Q}$ xraph}

\begin{tabular}{|l|l|l|l|l|l|}
\hline Close & Hdcpy & About \\
\hline
\end{tabular} Throughpu.

Throughput v/s Time

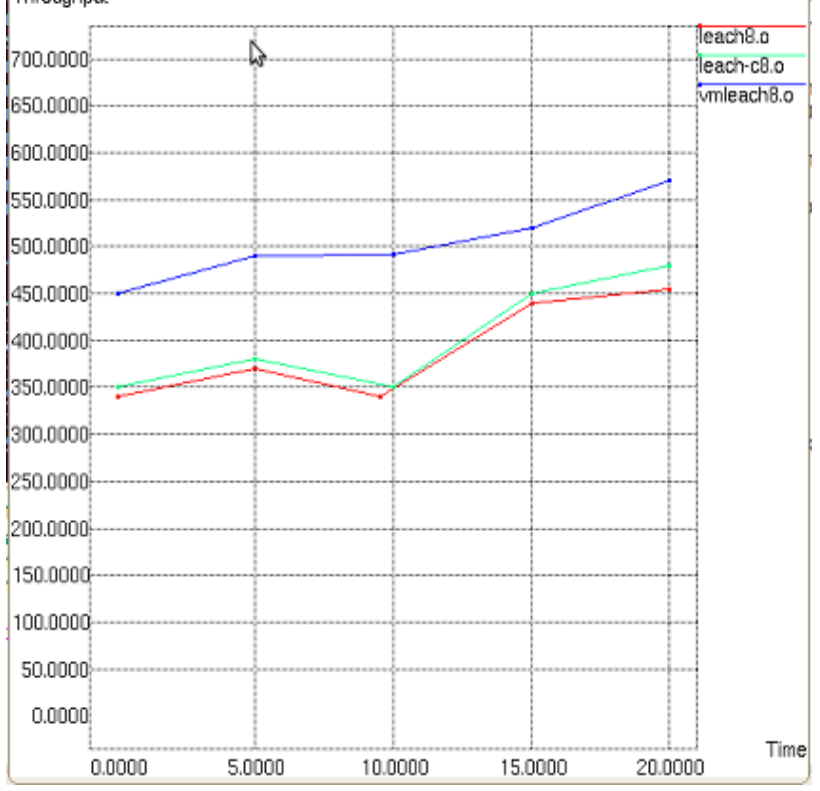

Fig 3: Throughput Vs Time

Generally throughput defines the average of successfully delivering data packets in a network. Fig. 3 shows that as time increases throughput also increases. VM LEACH provides high throughput compare to LEACH and LEACH C.

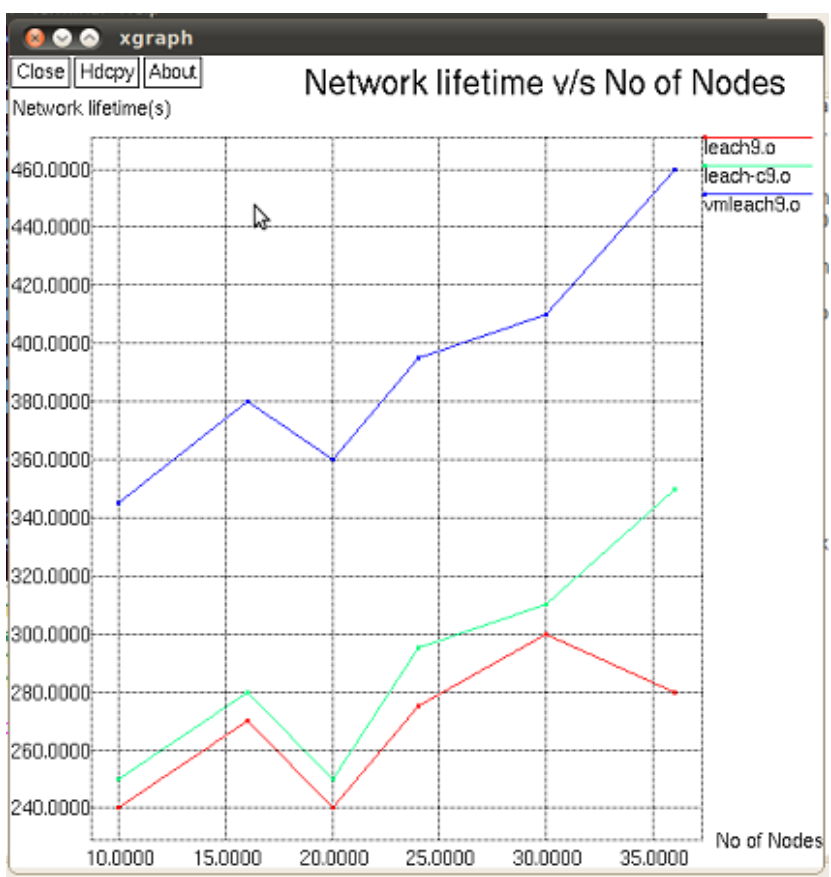

Fig 4: Network lifetime Vs number of nodes

The nodes use less energy, then the lifetime of nodes in a network wills increases. Fig. 4 shows by using variable round time with mobile collector leads the nodes to spend the less energy and increases the life time of the nodes. Overall network efficiency is been increased when compare to existing protocols. The MDC speed increases then delivery ratio also increases in a network.Fig.5 VM LEACH provides a good data delivery ratio by comparing to existing protocols. 
QQ $\mathrm{xgraph}$

Close Hdcpy About Delivery Ratio v/s Mobile collector speed Data Delivery Ratio

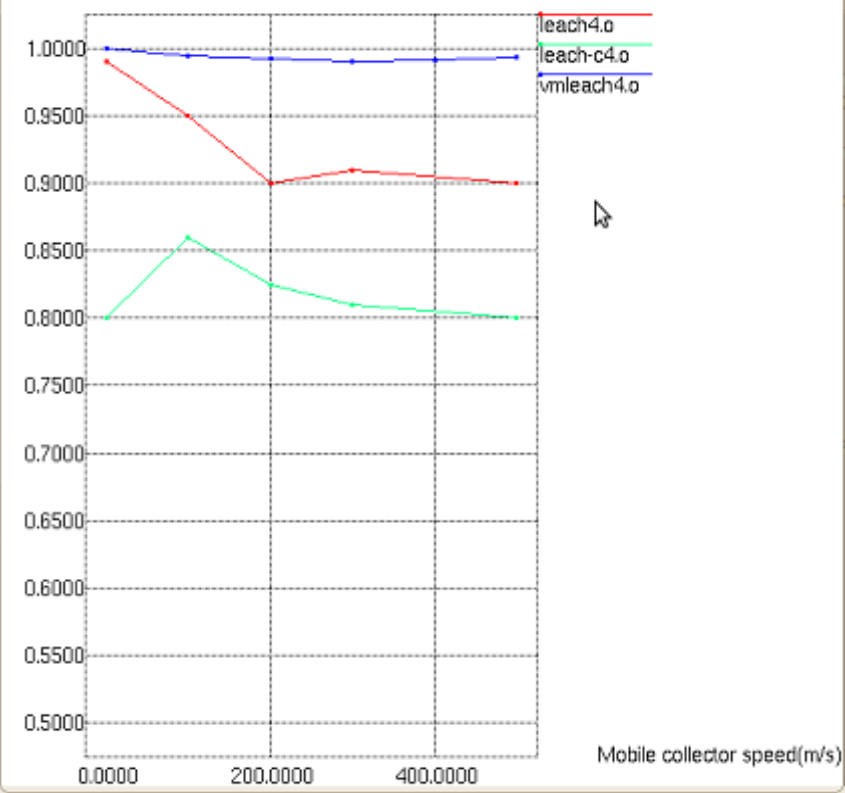

Fig 5: Data packet delivery ratio Vs MC speed

\section{CONCLUSION}

Proposed algorithm is an enhancement of clustering protocol by using an adaptive variable round time method with a mobile collector. The mobile collector provides a multi-hop communication between distance nodes to base station. It minimizes the energy consumption of nodes in the network, and can be used for larger geographical region Wireless Sensor Networks. The simulation studies reveals that the proposed VMLEACH gives us best performance in terms of energy. And also this algorithm compared with the existing routing protocols.

\section{REFERENCES}

[1] F. Akyildiz et al. "Wireless sensor networks: a survey", Computer Networks, Vol. 38, pp. 393-422, March 2002.

[2] Kemal Akkaya, Mohamed Younis, "Survey on routing protocols for wireless sensor networks Kemal”, ELSEVIER Ad hoc Networks 3(2005) pp:325349.

[3] Lan Tien Nguyen, Xavier Defago, Razvan Beuran, "An Energy Efficient Routing Scheme for Mobile Wireless Sensor Networks", IEEE/ international symposium on wireless communication system.2008, 'ISWCS '08'. Pages: 568-572.

[4] Houda Zeghilet, Nadjib Badache, Moufida Maimour, "Energy Efficient Cluster-based Routing in Wireless Sensor Networks", IEEE symposium on computers and communication 2009, ISCC- 2009. Pages: 701-704.

[5] Lindsey.s, Ragavendra C.S.," PEGASIS: Power-Efficient Gathering in Sensor Information Systems", IEEE/ Aerospace Conference proceedings,2002. Vol: 3, pp: 11251130.

[6] Sung-MinJung, Young-JuHan, Tai-MyoungChung, "The Concentric Clustering Scheme for Efficient Energy Consumption in the PEGASIS", IEEE/ International Conference on Advanced communication Technology.2007, Vol: 1, pp: 260-265.
[7] FengSen, QiBing, TangLiangrui, "An Improved EnergyEfficient PEGASIS-Based Protocol in Wireless Sensor Networks", IEEE/ International Conference on Fuzzy System and knowledge Discovery (FSFD), 2011. Vol:4, pp:2230-2233.

[8] Arati Manjeshwar, Dharma P. Agrawal, "TEEN: A Routing Protocol for Enhanced Efficiency in Wireless Sensor Networks", IEEE/ International proceedings parallel and distributed processing symposium 2000. pp: 2009-2015.

[9]Arati Manjeshwar, Dharma,P Agrawal, "APTEEN: A Hybrid Protocol for Efficient Routing and Comprehensive Information Retrieval in Wireless Sensor Networks", IEEE/ International proceedings on parallel and Distributed processing symposium(IPDPS) 2002.pp:195-202.

[10] SivaD.Muruganathan, Abraham O.Fapojuwo, "A Hybrid Routing Protocol for Wireless Sensor Networks Based on a Two-Level Clustering Hierarchy with Enhanced Energy Efficiency", IEEE/ Wireless communication and networking conference, WCNC-2008, pp.2051-2056.

[11] D. Baghyalakshmi, Jemimah Ebenezer, S.A.V. Satyamurty, " Low Latency And Energy Efficient Routing Protocols For Wireless Sensor Networks", IEEE/ International conference on wireless communication and sensor computing, ICWCSW2010. pp:1-6.

[12] OssamaYounis, Sonia Fahmy, "HEED: A Hybrid, EnergyEfficient, Distributed Clustering Approach for Ad Hoc Sensor Networks", IEEE Transactions On Mobile Computing, Vol. 3, No. 4, October-December 2004.

[13] W. Heinzelman, A.Chandrakasan, H. Balakrishnan, "An application specific protocol architecture for wireless micro sensor networks", IEEE Transaction on Wireless Networking, vol. 1, no. 4, pp. 660-670, Oct. 2002.

[14] Zhiyong PENG and Xiaojuan LI, "The Improvement and Simulation of LEACH Protocol for WSNs", IEEE/international conference on software engineering and service sciences (ICSESS), 2010. Pages: 500-503.

[15] Ahmed Ghneimat, John Mellor, Ping Jiang, "Adaptive, Cluster Based, Sensor Network Routing Protocol", IEEE/international conference on computer modelling and simulation (UKSim),2011. Pages: 472-476.

[16]Fan Xiangning, Song Yulin, "Improvement on LEACH Protocol of Wireless Sensor Networks", IEEE/ international conference on technologies and applications 2007, sensor comm. 2007. Pages: 260-264.

[17] N.Sandeep Krishna and C.Rajendra, "Refuse of Sensor's Energy Consumption in Wireless Sensor Networks Using Routing Protocols", International Journal of Advanced Research in Computer Engineering \& Technology Vol.1, No 4(2012.

[18] Vipin Pal, Girdhari Singh, and R P Yadav, "Analysing the Effect of Variable Round Time for Clustering Approach in Wireless Sensor Networks", LNSE 2013 Vol.1:pp 31-34 ISSN:2301-3559.

[19] Zhao, Fuzhexu, you;Li,Ru; Zhang, Wei, "Improved Leach Communication Protocol For WSN', IEEE/ International conference on Control Engineering and Communication Technology ICCECT-2012, pages:700-702.

[20] M. Bani Yassein, A. Al-zou'bi, Y. Khamayseh, W. Mardini, "Improvement on LEACH Protocol of Wireless Sensor Networks", International Journal of Digital Content Technology and its Applications, vol.3, num.2 ,June2009. 
[21] Ming Ma and Yuanyuan Yang, "Data Gathering in Wireless Sensor Networks with Mobile Collectors", IEEE/ International symposium on parallel and Distributed processing,2008. IPDPS-2008. Pages:1-9.

[22] Muhammad Arshad, Nasrullah Armi, Nidal Kamel and N. M. Saad, "Mobile data collector based routing protocol for wireless sensor networks", scientific Research and Essays Vol.6(29),pp.6162-6175,30 Nov,2011.

[23] Kumar A.K, Sivalingam K.M, "Energy-Efficient Mobile Data Collection in Wireless Sensor Networks with Delay Reduction using Wireless Communication", IEEE/
International conference on communication system and network, (COMSNETS),2010. Pages:1-10.

[24] Hisham M. Almasaeid and Ahmed E. Kamal, "Modelling Mobility-Assisted Data Collection in Wireless Sensor Networks",IEEE/ Global Telecommunication Conference GLOBECom-2008.pages:1-5.

[25] Mario Di Francesco and Sajal K. Das Crewman, Giuseppe Anastasi, "Data Collection in Wireless Sensor Networks with Mobile Elements: A Survey", ACM Transaction on sensor networks (TOSN) Vol.8 issue.1,Aug 2011. 